\title{
Efecto de un Pretratamiento Químico en el Deshidratado del Arándano por Métodos Combinados
}

\author{
Betty A. Ronceros, Roberto A. Quevedo y Javier I. Leiva \\ Universidad de Los Lagos, Departamento de Ciencia y Tecnología de los Alimentos, \\ Avenida Fuchslocher 1305, Osorno-Chile (e-mail: bronceros@ulagos.cl)
}

\begin{abstract}
Resumen
El objetivo de este trabajo fue estudiar la remoción química de piel de arándano a diferentes concentraciones de soda y tiempos de inmersión. La metodología utilizada emplea un diseño factorial de superficie de respuesta del tipo $2^{2}$. Como respuesta, se consideró la remoción de piel de arándano, ya sea total o parcial. Las variables analizadas fueron la concentración de soda y el tiempo de inmersión. Los resultados indicaron que la concentración de soda tuvo un efecto positivo marcado, al igual que el tiempo de inmersión pero en menor grado. Ambas variables no deben ser interpretadas separadamente, en razón del gran efecto de interacción entre ellas. Se concluye que al utilizar $1.5 \% \mathrm{NaOH}$ por 5 segundos, se obtiene una adecuada remoción de piel, sin dañar los frutos.
\end{abstract}

Palabras claves: arándanos, deshidratación osmótica, remoción química de piel, diseño factorial

\section{Effect of a Chemical Pretreatment on Blueberry Dehydrated Using Combined Methods}

\begin{abstract}
The objective of this paper was to study the chemical removal of blueberry skin at different soda concentrations and immersion times. In methodology, a surface response factorial design of type $2^{2}$ was considered. As a response, blueberry skin removal was considered, either total or partial. The variables analyzed were soda concentration and immersion time. Results indicated that soda concentration had a marked positive effect, as did immersion time although to a lesser extent. Both variables should not be interpreted separately, given the considerable effect produced by their interaction. It can be concluded that adequate skin removal was achieved by using $1.5 \% \mathrm{NaOH}$ for 5 seconds, without damaging the fruits.
\end{abstract}

Keywords: blueberries, osmotic dehydration, chemical skin removal, factorial design 


\section{INTRODUCCIÓN}

El aumento del consumo de arándanos a partir de la década del 90, junto a los requerimientos mundiales para economizar recursos, han llevado a la agroindustria a aplicar métodos mínimos de conservación para obtener alimentos de características similares a los frescos y que demanden menos energía para extender la vida útil (Panagiotou et al., 1998; Moraga et al., 2006). Una alternativa de conservación, es el desarrollo de un producto tipo pasa, deshidratado y dulce a partir del fruto del arándano (Heng et al., 1990; Álvarez et al., 1995).

Escriche et al. (2000), comentan que la etapa limitante en la deshidratación de frutos es el flujo de agua a través de la piel. Los mismos investigadores, indican que la remoción química de la piel facilita la salida de agua durante la deshidratación y la absorción controlada de azúcar entrega productos de mejor textura y aceptabilidad sensorial. Además, se mejora la rapidez en el secado, lo que da lugar a un mejor color en el fruto seco. Shi et al. (1997), estudiando el deshidratado de tomates, indicaron que $\mathrm{NaOH}$ mezclado con etil oleato produce menos daño en la piel y un retiro más alto de agua, que sólo una solución de $\mathrm{NaOH}$. Sin embargo, la solución de $\mathrm{NaOH}$ resultó más eficaz que $\mathrm{HCl}$ diluido en retiro de agua.

La metodología consiste en poner en contacto el fruto con la solución alcalina durante un periodo de tiempo controlado, determinando visualmente el tiempo óptimo para separar el fruto del reactivo químico (Cohen y Yang, 1995; Shi et al., 1997).

Investigaciones recientes han reportado condiciones óptimas de procesamiento para frutos mediante métodos mínimos. Shi et al. (1997), sugieren utilizar $\mathrm{NaOH}$ de concentración menor a 7\% para la obtención de una textura deseable en el fruto; Stojanovic y Silva (2007) junto con Doymaz (2006), aconsejan parámetros, como tiempo de osmoconcentración: $3 \mathrm{~h}$, temperatura de aire: $60^{\circ} \mathrm{C}$ y tiempo de secado: $25 \mathrm{~h}$.

El objetivo de este trabajo fue estudiar la remoción química de piel de arándano a diferentes concentraciones de soda y tiempos de inmersión.

\section{MATERIALES Y MÉTODOS}

Materia prima

Se utilizó arándanos (Vaccinium corymbosum) de la variedad Elliot cultivados en la provincia de Osorno (Chile), provenientes del descarte por tamaño de fruta fresca de exportación.

\section{Reactivo}

Se utilizó lentejas de $\mathrm{NaOH}$ (Merck). Las soluciones alcalinas se prepararon a temperatura de ebullición.

\section{Diseño factorial}

Se utilizó un diseño factorial de superficie de respuesta del tipo $2^{2}$ (Montgomery, 2002). El número total de tratamientos $(T)$ fue de 4, con dos puntos centrales. Cada tratamiento se realizó en duplicado y en forma aleatoria. Como respuesta se consideró la remoción, ya sea total o parcial, de piel de arándanos, los cuales fueron cuantificados como unidades defectuosas (D, expresado en \%). Las variables analizadas fueron la concentración de soda (C, expresado en \%) y el tiempo de inmersión (t, expresado en s). Los niveles de concentración fueron 1.5 y 4\%, con tiempos de inmersión de 5 y 15s (Tabla 1).

\section{Análisis de resultados}

El análisis de las respuestas D contempló: gráfico normal de probabilidad, análisis de varianza, análisis de regresión múltiple, análisis de correlación y superficie de respuesta. Los tratamientos se 
estudiaron con la técnica de análisis de componentes principales (Cuadras, 1991). Los programas computacionales utilizados fueron Statistica 6.0 y Statgraphics 4.0 (Statgraphics, 1994).

Tabla1: Planeamiento factorial para evaluar la remoción de piel

\begin{tabular}{|c|c|c|}
\hline \multirow{2}{*}{$\begin{array}{c}\text { Tratamientos } \\
\text { T }\end{array}$} & \multicolumn{2}{|c|}{ Variables } \\
\cline { 2 - 3 } & 1.5 & $\mathrm{t}$ \\
\hline 1 & 4 & 5 \\
3 & 1.5 & 5 \\
4 & 4 & 15 \\
5 & 2.75 & 15 \\
6 & 2.75 & 10 \\
\hline
\end{tabular}

\section{RESULTADOS Y DISCUSIÓN}

Las respuestas obtenidas en cada tratamiento se recogen en la Tabla 2. El análisis exploratorio de los datos mediante gráfico normal de probabilidad, estableció que el conjunto de respuestas (D) se ajustó razonablemente a una distribución normal (Pérez, 2004; Triola, 2004), lo que permite pensar en la existencia de normalidad y simetría en la distribución del conjunto de respuestas. En el eje de abscisas se ordenaron los valores $\mathrm{D}$, y en el eje de ordenadas las frecuencias relativas acumuladas de dichos valores (Figura 1).

Tabla 2: Respuestas D durante la remoción de piel

\begin{tabular}{|c|c|}
\hline Tratamiento & Respuesta \\
$\mathrm{T}$ & $\mathrm{D}(\%)$ \\
\hline 1 & 1.85 \\
2 & 13.21 \\
3 & 7.84 \\
4 & 46.15 \\
5 & 16.67 \\
6 & 17.58 \\
\hline
\end{tabular}

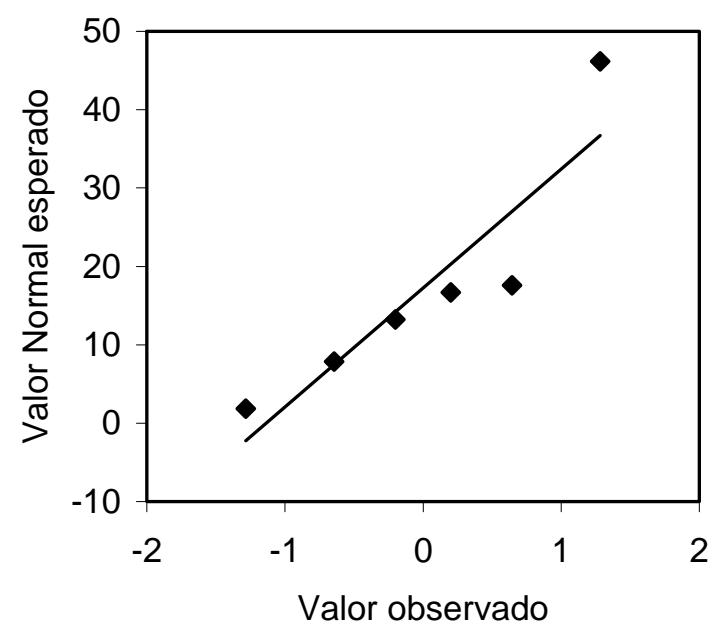

Fig. 1: Gráfico normal de probabilidad de arándanos defectuosos.

En la Tabla 3 se muestran los efectos principales y de interacción, cuando el valor $D$ varió de un nivel bajo a uno alto para cualquiera de las variables: C y t. La desviación estándar se basó en un error total con 2 grados de libertad. 
Tabla 3: Efectos principales y de interacción para las variables analizadas durante la remoción de piel

\begin{tabular}{|c|c|c|}
\hline Efecto & Valor & Desviación estándar \\
\hline $\mathrm{C}$ & +24.83 & \pm 0.46 \\
$\mathrm{t}$ & +19.46 & \pm 0.46 \\
$\mathrm{Ct}$ & +13.47 & \pm 0.46 \\
\hline
\end{tabular}

La concentración de soda ejerció un efecto positivo marcante $(+24)$ y el tiempo de inmersión tiene un efecto sensible en el mismo sentido pero en menor grado (+19). El efecto de interacción indica que ambas variables no pueden ser interpretadas separadamente, en razón del gran efecto de interacción entre ellas (+13).

En la Tabla 4 se muestran los resultados del ANOVA para las variables en estudio. Existió diferencia significativa, confirmándose así el efecto significativo de cada variable y su interacción. Los coeficientes de determinación fueron satisfactorios $\left(r^{2}=0.99962\right.$, y ajustado para los grados de libertad: $\left.r^{2}=0.99906\right)$, observándose un bajo error.

Tabla 4: ANOVA para las variables analizadas durante la remoción de piel

\begin{tabular}{|c|r|r|r|r|}
\hline $\begin{array}{c}\text { Fuente de } \\
\text { variación }\end{array}$ & $\begin{array}{c}\text { Grados de } \\
\text { libertad }\end{array}$ & $\begin{array}{c}\text { Suma de } \\
\text { cuadrados }\end{array}$ & \multicolumn{1}{|c|}{$\mathrm{F}_{\text {experimental }}$} & \multicolumn{1}{c|}{$\mathrm{F}_{\text {tabla }}$} \\
\hline $\mathrm{C}$ & 1 & 616.77 & 2808.27 & 0.0004 \\
$\mathrm{t}$ & 1 & 378.88 & 1725.12 & 0.0006 \\
$\mathrm{Ct}$ & 1 & 181.57 & 826.74 & 0.0010 \\
Error total & 2 & 0.43 & - & - \\
\hline
\end{tabular}

En un estudio realizado para optimizar la extracción de jugo de sapodilla con agua caliente, Sin et al. (2006) concluyeron que la temperatura fue el factor que más afectó la extracción y las características del jugo. Los mismos investigadores, indicaron que las condiciones óptimas para realizar la extracción fueron de $60^{\circ} \mathrm{C}$ por $120 \mathrm{~min}$.

El análisis de regresión múltiple permitió formular un modelo matemático (Ec. 1) para predecir el número de frutos defectuosos bajo las condiciones experimentales utilizadas en la investigación. Se observó un bajo error $\left(r^{2}=0.9996\right)$. El modelo desarrollado fue validado con los datos experimentales mostrando como las condiciones de operación: concentración de soda y tiempo de inmersión, afectan la respuesta. Modelos de regresión similares, describiendo el deshidratado en plátanos y melones han obtenido Fernandes et al. (2006) y Teles et al. (2006).

$D=0.078-0.846 C-1.018 t+1.078 C t$

Siguiendo la metodología descrita por Sin et al. (2006) para el estudio de modelos de regresión en alimentos, se utilizó la matriz de correlación para analizar la relación entre las variables independientes y la respuesta, estimándose correlaciones positivas y significativas a un nivel de $(P<0,05)$ entre: C y $D$, y entre t y $D$. Se encontró una asociación más estrecha entre C y D (Tabla 5).

Tabla 5: Matriz de correlación para las variables analizadas durante la remoción de piel

\begin{tabular}{|c|c|c|c|}
\hline & $C$ & $t$ & $D$ \\
\hline$C$ & & & \\
$t$ & -0.00 & & \\
$D$ & 0.72 & 0.56 & \\
\hline
\end{tabular}

La respuesta $D$ fue analizada mediante la metodología de superficie de respuesta para analizar por simultaneo el efecto de las variables $\mathrm{C}$ y $\mathrm{t}$ (Figura 2). Los resultados indicaron que los frutos tratados con una alta concentración de soda (4.0\%), en los niveles mínimo y máximo del tiempo de inmersión, presentaron un alto grado de remoción de piel. A su vez, al utilizar una menor concentración de soda (1.5\%), la respuesta fue menor. Los resultados encontrados fueron concordantes con la investigación 
realizada por Shi et al. (1997), con respecto a la remoción de piel en tomate deshidratado. Sato et al. (2006), encontraron que al aumentar la temperatura de la solución tratante, se promueven cambios en el color del producto final, haciendo a la fruta más oscura. Los mismos autores sugieren adicionar calcio para promover un mejoramiento de la textura y minimizar los efectos negativos de la temperatura en las propiedades mecánicas del fruto.

La representación gráfica de acuerdo a componentes principales se indica en la Figura 3 (Cuadras, 1991); en ella, se observó en un plano cartesiano, la ubicación de los 6 tratamientos, y las 2 variables analizadas junto con la respuesta estudiada utilizando factores como ejes de abscisas y ordenadas. En el primer y cuarto cuadrante, se ubicaron las variables $C$ y t, y la respuesta D. Por su parte, los tratamientos T2, T5 y T6 se concentraron cercanos a la concentración y en menor grado al tiempo, en particular los dos últimos.

Según Philippeau (1990), estos resultados indican que los tratamientos T5 y T6 tienen una estrecha asociación con la concentración de soda y una menor relación con el tiempo de inmersión durante la remoción de piel. Además, el tratamiento T4, se relaciona con la respuesta $D$, por lo que se sugiere su selección cuando el daño superficial en el arándano sea un criterio de interés. En la representación gráfica de los tratamientos destaca la ubicación opuesta del tratamiento T1 y T3 con la respuesta $\mathrm{D}$ y las variables concentración y tiempo.

El efecto de la respuesta en cada tratamiento se indica en la Figura 4. En cuanto a la fracción de frutos defectuosos, éstos presentaron una tendencia superior para el caso de T3, T4, T5 y T6, especialmente el primero de ellos. De acuerdo con los resultados obtenidos, se sugiere la selección de los tratamientos indicados anteriormente a la hora de ejecutar un experimento que busque el deterioro controlado en la superficie del fruto.

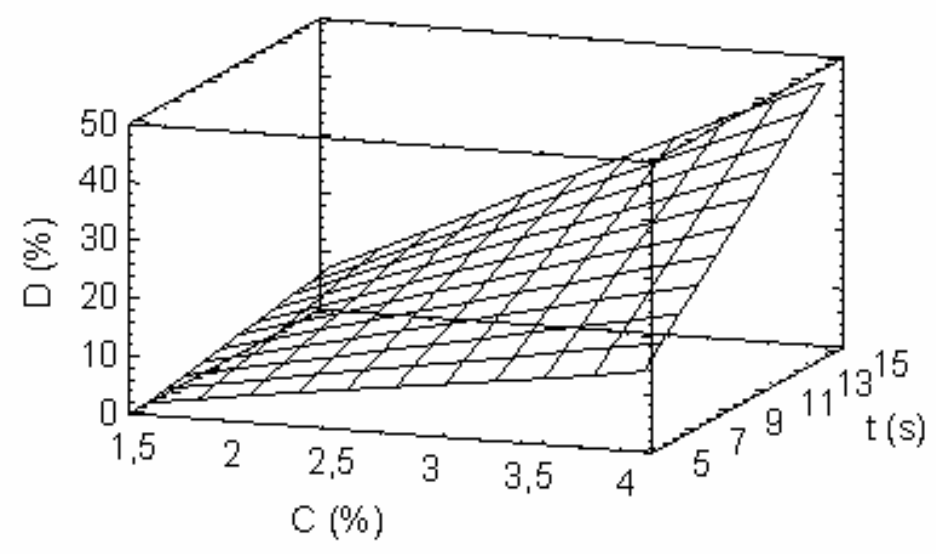

Fig. 2: Superficie de respuesta de arándanos con remoción de piel.

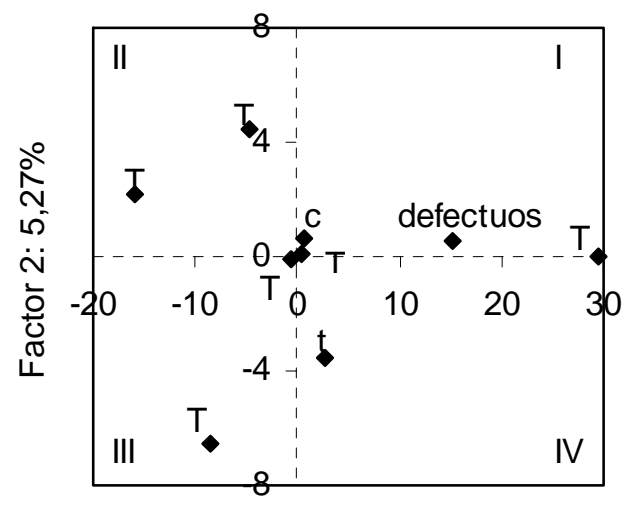

Factor 1: 94,62\%

Fig. 3: Representación de los tratamientos, variables y respuesta en los ejes factoriales 1 y 2. 


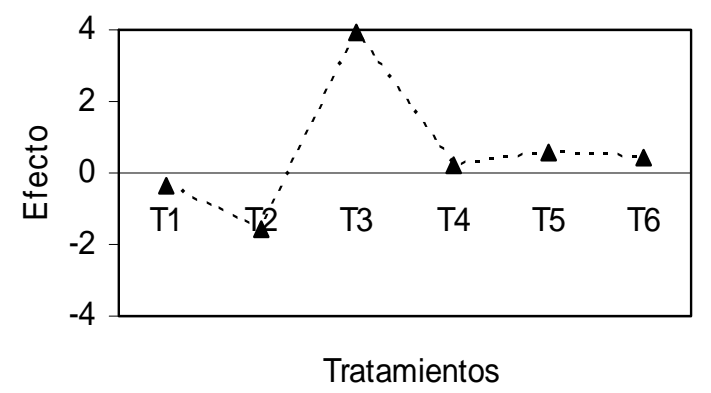

Fig. 4: Efecto de la respuesta en cada tratamiento de remoción de piel.

\section{CONCLUSIONES}

Para la obtención de frutos con mínima remoción de piel, se recomienda emplear soda al 1.5\% y 5s como tiempo de inmersión. Para una remoción intensiva de piel: $4 \% \mathrm{NaOH}$ y 15s.

La remoción química de piel, puede utilizarse como una tecnología para aprovechar frutos descartados por tamaño, siendo necesaria la aplicación de un método combinado de deshidratado osmótico seguido por un secado con aire caliente.

El modelo propuesto para interpretar el fenómeno de remoción de piel en arándano, permite tener en cuenta las variables: concentración de soda y tiempo de inmersión. Las predicciones del modelo se ajustaron adecuadamente a los datos experimentales, pudiendo ser una herramienta útil para interpretar el mismo fenómeno en otros berries.

\section{AGRADECIMIENTOS}

Los autores agradecen a Susan Angus por su colaboración en la traducción del resumen. Esta investigación se desarrolló con apoyo financiero de CORFO (Proyecto Fontec \#203-3822).

\section{REFERENCIAS}

Álvarez, C. y otros cinco autores; Air dehydration of strawberries-effects of blanching and osmotic pretreatments on the kinetics of moisture transport, J. Food Eng., 25(2), 167-178 (1995).

Cohen, J.S y T.C. Yang; Progress in food dehydration, Trends Food Sci. Tech., 6(1), $20-25$ (1995).

Cuadras, C.; Métodos de análisis multivariante, Colección estadística y análisis de datos, Barcelona, España (1991).

Doymaz, I.; Drying kinetics of black grapes treated with different solutions, J. Food Eng., 76(2), 212217 (2006).

Escriche, I., A. Chiralt, J. Moreno y J. Serra; Influence of blanching-osmotic dehydration treatments on volatile fraction of srtawberries, J. Food Sci., 65(7), 1107-1111 (2000).

Fernandes, F.A., S. Rodrigues, O.C. Gaspareto y E.L. Oliveira; Optimization of osmotic dehydration of bananas followed by air-drying, J. Food Eng., 77(1), 188-193 (2006).

Heng, K., S. Guilbert y J. Cuq; Osmotic dehydration of papaya-influence of process variables on the product quality, Science des Aliments, 10(4), 831-848 (1990).

Montgomery, D.C.; Diseño y análisis de experimentos, Editorial Iberoamericana, México D.F. (2002).

Moraga, G., N. Martinez-Navarrete y A. Chiralt; Compositional changes of strawberry due to dehydratation, cold storage and freezingthawing processes, J. Food Process Pres., 30(4), 458-474 (2006). 
Panagiotou, N., V. Karathanos y Z. Maroulis; Mass transfer modelling of the osmotic dehydratation of some fruits, Int. J. Food Sci. Tech., 33(3), 267-284 (1998).

Pérez, C.; Técnicas de Análisis Multivariante de Datos, Pearson Prentice Hall, Madrid, España (2004).

Philippeau, G.; ¿Cómo interpretar los resultados de un análisis de componentes principales?, Service dus Eludes Statistiques, Francia (1990).

Sato, A.C., E.J. Sanjinez-Argandona y R.L. Cunha; The effect of addition of calcium and processing temperature on the quality of guava in syrup, Int. J. Food Sci. Tech., 41(4), 417-424 (2006).

Shi, J.X., M. Le Maguer, S.L. Wang y A. Liptay; Application of osmotic treatment in tomato processing-effect of skin treatments on mass transfer in osmotic dehydration of tomatoes, Food Res. Int., 30(9), 669-674 (1997).

Sin, H.N., S. Yusof, N.S. Hamid y R.A. Rahman; Optimization of hot water extraction for sapodilla juice using response surface methodology, J. Food Eng., 74(3), 352-358 (2006).

Statgraphics Plus. Version 7.1; Users guide reference, 63-73, Statistical graphics, México D.F. (1994).

Stojanovic, J. y J.L. Silva: Influence of osmotic concentration, continuous high frequency ultrasound and dehydration on antioxidants, colour and chemical properties of rabbiteye blueberries, Food Chem., 101(3), 898-906 (2007).

Teles, U.M. y otros cinco autores; Optimization of osmotic dehydration of melons followed by airdrying, Int. J. Food Sci. Tech., 41(6), 674-680 (2006).

Triola, M.F.; Estadística, Pearson Addison Wesley, Naucalpan de Juárez, México D.F. (2004). 
